\title{
DIRECT-SUM DECOMPOSITION OF ATOMIC AND ORTHOGONALLY COMPLETE RINGS
}

\author{
ALEXANDER ABIAN
}

(Received 5 March 1969)

Communicated by G. B. Preston

In this paper we give a necessary and sufficient condition for decomposition (as a direct sum of fields) of a ring $R$ in which for every $x \in R$ there exists a (and hence the smallest) natural number $n(x)>1$ such that

$$
x^{n(x)}=x .
$$

We would like to emphasize that in what follows $R$ stands for a ring every element $x$ of which satisfies (1).

It is well known [1] that $R$ is commutative and that $x^{n(x)-1}$ is an idempotent element of $R$, i.e., for every $x \in R$

$$
\left(x^{n(x)-1}\right)^{2}=x^{n(x)-1}
$$

which implies that $R$ has no nonzero nilpotent element, i.e., for every $x \in R$ and every natural number $k \geqq 1$,

$$
x^{k}=0 \text { implies } x=0 .
$$

LEMMA 1. The ring $R$ is partially ordered by $\leqq$ where for all elements $x$ and $y$ of $R$

$$
x \leqq y \text { if and only if } x y=x^{2} .
$$

Proof. Since $x x=x^{2}$ we see that $\leqq$ is reflexive.

Next, let $x \leqq y$ and $y \leqq x$, i.e. $x y=x^{2}$ and $y x=y^{2}$. But then

$$
x^{2}-x y-y x-y^{2}=(x-y)^{2}=0
$$

which, in view of (3), implies $x-y=0$, i.e. $x=y$. Hence $\leqq$ is antisymmetric.

Finally, let $x \leqq y$ and $y \leqq z$, i.e., $x y=x^{2}$ and $y z=y^{2}$. Thus, $x^{2} z=x y z=$ $x y^{2}=x^{2} y=x^{3}$. Consequently, $x^{2} z^{2}=x^{3} z$ and $x^{3} z=x^{4}$. But then

$$
x^{2} z^{2}-2 x^{3} z+x^{4}=\left(x z-x^{2}\right)^{2}=0
$$

which, in view of (3), implies $x z=x^{2}$ which in turn, in view of (4), implies $x \leqq z$. Hence $\leqq$ is transitive.

Thus, Lemma 1 is proved. 
Clearly, from (4) and (2) it follows that for all elements $x, y$ and $z$ of $R$

$$
y \leqq z \text { implies } x y \leqq x z
$$

and

$$
x^{n(x)-1} y \leqq y .
$$

Definition 1. A nonzero element a of $R$ is called an atom of $R$ provided for every $x \in R$

$$
x \leqq a \text { implies } x=a \text { or } x=0 .
$$

Moreover, $R$ is called atomic provided for every nonzero element $r$ of $R$ there exists an atom $a$ of $R$ such that $a \leqq r$.

LEMMA 2. Let $a$ be an atom of $R$. Then

$$
r^{n(r)-1} a=a \text { or } \quad r a=0
$$

for every element $r$ of $R$.

Proof. By (6) we have $r^{n(r)-1} a \leqq a$ and since $a$ is an atom, by (7) we have $r^{n(r)-1} a=a$ or $r^{n(r)-1} a=0$. However, $r^{n(r)-1} a=0$ in view of (1) implies $r a=0$.

Definition 2. A subset $S$ of $R$ is called orthogonal provided $x y=0$ for distinct elements $x$ and $y$ of $S$.

LEMMA 3. The set $\left(e_{i}\right)_{i \in I}$ of all idempotent atoms of $R$ is an orthogonal set.

Proof. Since each $e_{i}$ is both an atom and an idempotent, from Lemma 2 it follows that $e_{i} e_{j}=e_{i}=e_{j}$ or $e_{i} e_{j}=0$.

LEMMA 4. Let a be an atom of $R$. Then $a^{n(a)-1}$ is an idempotent atom of $R$.

Proof. From (2) it follows that $a^{n(a)-1}$ is idempotent.

Now, let $x \leqq a^{n(a)-1}$. But then (5) and (1) imply $a x \leqq a$. Since $a$ is an atom (7) implies $a x=a$ or $a x=0$.

If $a x=a$ then $a^{n(a)-1} x=a^{n(a)-1}$ which by (4) implies $a^{n(a)-1} \leqq x$. Hence $x=a^{n(a)-1}$.

If $a x=0$ then $a^{n(a)-1} x=0$; but $a^{n(a)-1} x=x^{2}$ by definition of $\leqq$, therefore $x^{2}=0$ which by (3) implies $x=0$.

LemMa 5. Let $\left(e_{i}\right)_{i \in I}$ be the set of all idempotent atoms of $R$. Then for every $i \in I$ the ideal $F_{i}$ of $R$ given by

$$
F_{i}=\left\{r e_{i} \mid r \in R\right\}
$$

is a subfield of $R$. Moreover,

$$
F_{i} \cap F_{j}=\{0\} \text { if } i \neq j .
$$


Proof. Since $e_{i}^{2}=e_{i}$ it follows that $e_{i}$ is an element of $F_{i}$ and also the unit of $F_{i}$.

Now, let $r e_{i} \neq 0$. We show that $r e_{i}$ has an inverse in $F_{i}$. If $n(r)=2$ then Lemma 2 implies $r e_{i}=e_{i}$ which shows that $r e_{i}$ is its own inverse in $F_{i}$. If $n(r)>2$ then Lemma 2 implies $\left(r e_{i}\right)\left(r^{n(r)-2} e_{i}\right)=e_{i}$ which shows that $r^{n(r)-2} e_{i}$ is the inverse of $r e_{i}$ in $F_{i}$.

Next, if $i \neq j$ and $r e_{i}=q e_{j}$ for $r, q \in R$ then Lemma 3 implies $r e_{i} e_{j}=q e_{j}=$ $r e_{i}=0$.

LEMMA 6. Let $R$ be atomic and let $\left(e_{i}\right)_{i \in I}$ be the set of all idempotent atoms of $R$. Then for every nonzero element $q$ of $R$ there exists an idempotent atom, say, $e_{k}$ such that $q e_{k} \neq 0$. Moreover, for every $r \in R$ the $\sup _{i} r e_{i}$ exists and

$$
r=\sup _{i} r e_{i}
$$

Proof. In view of (7) there exists an atom $a$ such that $a \leqq q$, i.e., $a q=a^{2} \neq 0$. But then Lemma 4 and (1) imply that $e_{k}=a^{n(a)-1}$ is an idempotent atom and $a^{n(a)-1} q=a^{n(a)}=a \neq 0$, i.e., $q e_{k} \neq 0$.

Next, since $r e_{i}=\left(r e_{i}\right)^{2}$ for every $i \in I$, it follows that $r$ is an upper bound of $\left(r e_{i}\right)_{i \in I}$. Let $h$ be any upper bound of $\left(r e_{i}\right)_{i \in I}$, i.e. $h r e_{i}=\left(r e_{i}\right)^{2}$ for every $i \in I$. We show that $r \leqq h$. Because otherwise, $h r-r^{2}=q \neq 0$ and therefore $h r e_{k}-r^{2} e_{k}=q e_{k} \neq 0$, contradicting that $h r e_{i}=r r e_{i}$ for every $i \in I$.

Thus, Lemma 6 is proved.

Let us observe that if $\left(e_{i}\right)_{i \in I}$ is the set of all idempotent atoms of $R$ then in view of (9) we may consider the direct sum $\oplus_{i \in I} F_{i}$ of the fields $F_{i}$ given by (8). In this connection we have the following

LEMMA 7. Let $R$ be atomic and let $\left(e_{i}\right)_{i \in I}$ be the set of all idempotent atoms of $R$. Then

$$
\alpha(r)=\left(r e_{i}\right)_{i \in I}
$$

is an isomorphism from $R$ into the direct sum $\oplus_{i \in I} F_{i}$ of fields $F_{i}$.

Proof. It is obvious that $\alpha$ is a homomorphism. We show that $\alpha$ is one-toone. Indeed, if $r \neq q$ then $\alpha(r) \neq \alpha(q)$. Because otherwise, (10) would imply $r=\sup _{i} r e_{i}=\sup _{i} q e_{i}=q$, contradicting $r \neq q$.

Thus, Lemma 7 is proved.

Let us observe that the existence of an isomorphism from $R$ onto a subring of a direct sum of fields is a well known fact and is proved without imposing any special condition (such as atomicity) on $R$. However, for the proof of our Theorem we need (as seen below) the special isomorphism $\alpha$ described in Lemma 7. In fact the existence of the isomorphism $\alpha$ is crucial for the proof of our Theorem which states that atomicity and orthogonal completeness of $R$ is a necessary and sufficient 
condition for $R$ to be isomorphic to a direct sum of fields. The proof uses Lemma 8 below.

First however, we observe that if $\left(r_{i}\right)_{i \in I}$ is a subset of $R$ such that $\sup _{i} r_{i}$ exists then, since $r_{i} \leqq \sup _{i} r_{i}$, in view of (3) we have

$$
r_{i} \sup _{i} r_{i}=r_{i}^{2}
$$

LEMMA 8. Let $\left(r_{i}\right)_{i \in I}$ be a subset of $R$ such that $\sup _{i} r_{i}$ exists. Then for every element $b$ of $R$ the $\sup _{i} b r_{i}$ exists and

$$
b \sup _{i} r_{i}=\sup _{i} b r_{i} .
$$

Proof. In view of (12) we have

$$
\left(b r_{i}\right)\left(b \sup _{i} r_{i}\right)=b^{2} r_{i} \sup _{i} r_{i}=\left(b r_{i}\right)^{2}
$$

which, in view of (4), implies $b r_{i} \leqq b \sup _{i} r_{i}$ for every $i \in I$. Thus, $b$ sup $r_{i}$ is an upper bound of $\left(b r_{i}\right)_{i \in I}$.

Next, let $u$ be any upper bound of $\left(b r_{i}\right)_{i \in I}$, i.e., $b r_{i} \leqq u$, which, in view of (4), implies that for every $i \in I$,

$$
b r_{i} u-b^{2} r_{i}^{2}+r_{i}^{2}=r_{i}^{2} .
$$

But then from (12) it follows that for every $i \in I$

and therefore

$$
r_{i}\left(b u-b^{2} \sup _{i} r_{i}+\sup _{i} r_{i}\right)=r_{i}^{2}
$$

$$
r_{i} \leqq b u-b^{2} \sup _{i} r_{i}+\sup _{i} r_{i}
$$

which implies

$$
\sup _{i} r_{i} \leqq b u-b^{2} \sup _{i} r_{i}+\sup _{i} r_{i}
$$

But then from (4) it follows that

which yields

$$
\left(\sup _{i} r_{i}\right)\left(b u-b^{2} \sup _{i} r_{i}+\sup _{i} r_{i}\right)=\left(\sup _{i} r_{i}\right)^{2}
$$

$$
\left(b \sup _{i} r_{i}\right) u=\left(b \sup _{i} r_{i}\right)^{2}
$$

implying by (4) that $b \sup r_{i} \leqq u$. Hence $\left(b r_{i}\right)_{i \in I}$ has a supremum which is equal to $b \sup r_{i}$.

DEFINITION 3. The ring $R$ is called orthogonally complete provided $\sup S$ of every orthogonal subset $S$ of $R$ exists.

Finally, we prove: 
THEOREM. The ring $R$ is isomorphic to a direct sum of fields if and only if $R$ is atomic and orthogonally complete.

Proof. Let $\beta$ be an isomorphism from $R$ onto a direct sum $\oplus_{i \in I} K_{i}$ of fields $K_{i}$. Let $r$ be a nonzero element of $R$ and let $\beta(r)=\left(r_{i}\right)_{i \in I}$. Without loss of generality we may assume that $r_{1} \neq 0$. Let $u_{1}$ be the unit of $K_{1}$. But then $a=r \beta^{-1}\left(\left(k_{i}\right)_{i \in I}\right)$ with $k_{1}=u_{1}$ and $k_{i}=0$ for $i \neq 1$ is obviously an atom of $R$ such that $a \leqq r$. Thus, $R$ is atomic. Next, let $S$ be an orthogonal subset of $R$ and let $\beta[S]=\left(\left(k_{i}(s)\right)_{i \in I}\right)_{s \in S}$. But then, in view of the orthogonality of $S$, clearly, $\beta^{-1}\left(\left(k_{i}\right)_{i \in I}\right)=\sup S$ where $k_{i}=k_{i}(s)$ if $k_{i}(s) \neq 0$ for some $s \in S$, and, otherwise $k_{i}=0$. Thus, $R$ is orthogonally complete.

Conversely, we show that if $R$ is atomic and orthogonally complete then $R$ is isomorphic to the direct sum $\oplus_{i \in I} F_{i}$ of fields $F_{i}$ mentioned in Lemma 7. To this end we show that the isomorphism $\alpha$ mentioned in Lemma 7 is an onto mapping. Let $\left(r_{i} e_{i}\right)_{i \in I}$ be an element of $\oplus_{i \in I} F_{i}$. From Lemma 3 it follows readily that $\left(r_{i} e_{i}\right)_{i \in I}$ is an orthogonal subset of $R$. Let $h=\sup _{i} r_{i} e_{i}$. But then from (13) and Lemma 3 it follows that $h e_{j}=e_{j} \sup _{i} r_{i} e_{i}=r_{j} e_{j}$ for every $j \in I$. Hence $\left(h e_{j}\right)_{j \in I}=\left(r_{i} e_{i}\right)_{i \in I}$. However, from (11) it follows that $\alpha(h)=\left(h e_{j}\right)_{j \in I}=\left(r_{i} e_{i}\right)_{i \in I}$. Thus, $\left(r_{i} e_{i}\right)_{i \in I}$ is in the range of $\alpha$ and therefore $\alpha$ is an onto mapping, as desired.

\section{Reference}

[1] N. Jacobson, Structure of Rings, Amer. Math. Soc. Coll. Publ. Vol. 37 (1956), p. 217.

Iowa State University

Ames, Iowa 